\title{
UNIBRANCH ORBIT CLOSURES IN MODULE VARIETIES
}

\author{
BY GRZEGORZ ZWARA
}

ABSTRACT. - Let $A$ be a finite dimensional associative algebra over an algebraically closed field such that there are, up to isomorphism, only finitely many indecomposable left $A$-modules. We show that the orbit closures in the associated module varieties are unibranch.

(C) 2002 Éditions scientifiques et médicales Elsevier SAS

RÉSUMÉ. - Soit $A$ une algèbre associative avec unité, de dimension finie sur un corps algébriquement clos et telle que $A$ ne possède qu'un nombre fini de modules à gauche indécomposables, à isomorphisme près. Nous montrons que l'adhérence de toute orbite dans la variété associée de modules est unibranche.

(c) 2002 Éditions scientifiques et médicales Elsevier SAS

\section{Introduction}

Throughout the paper, $k$ denotes a fixed algebraically closed field and $A$ an associative finite dimensional $k$-algebra with identity. Furthermore, $\bmod A$ stands for the category of finite dimensional left $A$-modules. A variety means an algebraic reduced $k$-scheme and a point of a variety is always assumed to be closed.

Let $d \geqslant 1$ and denote by $\mathbb{M}_{d}(k)$ the algebra of $d \times d$-matrices with coefficients in $k$. The set $\bmod _{A}^{d}(k)$ of the $A$-module structures on the vector space $k^{d}$, i.e. algebra homomorphisms $M: A \rightarrow \mathbb{M}_{d}(k)$, has a natural structure of an affine variety. Moreover, the general linear group $G l_{d}(k)$ acts on $\bmod _{A}^{d}(k)$ by conjugation and the $G l_{d}(k)$-orbits correspond bijectively to the isomorphism classes of $d$-dimensional left $A$-modules. We shall denote by $\mathcal{O}_{M}$ the orbit in $\bmod _{A}^{d}(k)$ corresponding to a $d$-dimensional module $M$ in $\bmod A$. An interesting problem is to study geometric properties of orbit closures in $\bmod _{A}^{d}(k)$. It was proved in [1] that the orbit closures are normal Cohen-Macaulay varieties with rational singularities provided $A$ is the path algebra of a Dynkin quiver of type $\mathbb{A}_{n}$.

One of the methods to study the geometry of a variety $\mathcal{X}$ is to investigate a resolution of singularities of $\mathcal{X}$, that is a proper, birational morphism $\mathcal{Y} \rightarrow \mathcal{X}$ where $\mathcal{Y}$ is a smooth variety. Let $M \in \bmod A$. Our first aim is to construct a resolution of singularities of the orbit closure $\overline{\mathcal{O}}_{M}$. Recently M. Reineke has constructed a resolution of singularities for the orbit closures of representations of Dynkin quivers (see [5]).

Let $X \in \bmod A, d=\operatorname{dim}_{k} M$ and $c=[X, M]_{A}$. Here and later on,

$$
[Y, Z]_{A}=\operatorname{dim}_{k} \operatorname{Hom}_{A}(Y, Z)
$$

for any modules $Y, Z \in \bmod A$. We denote by $\operatorname{add}(X)$ the full subcategory of $\bmod A$ consisting of the modules isomorphic to a direct summand of $X^{i}$ for some $i \geqslant 1$. The canonical action of $\mathrm{Gl}_{d}(k)$ on the space $\operatorname{Hom}_{k}\left(X, k^{d}\right)$ induces canonically an action of $\mathrm{Gl}_{d}(k)$ on the Grassmann 
variety $\operatorname{Grass}\left(\operatorname{Hom}_{k}\left(X, k^{d}\right), c\right)$ of $c$-dimensional subspaces of the vector space $\operatorname{Hom}_{k}\left(X, k^{d}\right)$. We consider the $\mathrm{Gl}_{d}(k)$-variety

$$
\mathcal{C}=\bmod _{A}^{d}(k) \times \operatorname{Grass}\left(\operatorname{Hom}_{k}\left(X, k^{d}\right), c\right),
$$

and its one special $\mathrm{Gl}_{d}(k)$-orbit

$$
\mathcal{O}_{M_{X}}=\left\{\left(M^{\prime}, \operatorname{Hom}_{A}\left(X, M^{\prime}\right)\right) ; M^{\prime} \in \mathcal{O}_{M}\right\} .
$$

Here, $\operatorname{Hom}_{A}\left(X, M^{\prime}\right)$ is a subspace of $\operatorname{Hom}_{k}\left(X, k^{d}\right)$ consisting of the maps $f$ such that $f(a x)=M^{\prime}(a) f(x)$ for any $a \in A$ and $x \in X$. We first study properties of the restriction $p_{M, X}: \overline{\mathcal{O}}_{M_{X}} \rightarrow \overline{\mathcal{O}}_{M}$ of the canonical projection $\mathcal{C} \rightarrow \bmod _{A}^{d}(k)$.

THEOREM 1.1. - The morphism $p_{M, X}$ is projective and birational, and $\overline{\mathcal{O}}_{M_{X}}$ consists of the points $(N, V) \in \mathcal{C}$ such that there is an exact sequence

$$
0 \rightarrow Z \rightarrow Z \oplus M \stackrel{\beta}{\rightarrow} N \rightarrow 0
$$

in $\bmod A$ with $V=\operatorname{im}_{H^{\prime}}(X, \beta)$. Moreover, if $Z \oplus M \in \operatorname{add}(X)$ then $(N, V)$ is a smooth point of $\overline{\mathcal{O}}_{M_{X}}$.

Let ind $A$ be a complete set of pairwise nonisomorphic indecomposable modules in $\bmod A$. We denote by $\mathcal{I}(M)$ the set of $Y \in \operatorname{ind} A$ such that there is an injective $A$-homomorphism $Y \rightarrow M^{i}$ for some $i \geqslant 1$. Our main result is as follows.

THEOREM 1.2. - Let $M$ be a module in $\bmod A$ such that $\mathcal{I}(M)$ is a finite set. Then:

(1) $p_{M, X}: \overline{\mathcal{O}}_{M_{X}} \rightarrow \overline{\mathcal{O}}_{M}$ is a resolution of singularities if $\mathcal{I}(M) \subseteq \operatorname{add}(X)$;

(2) the fibres of $p_{M, X}$ are connected;

(3) $\overline{\mathcal{O}}_{M}$ is a unibranch variety;

(4) $\overline{\mathcal{O}}_{M}=\left\{N \in \bmod _{A}^{d}(k) ;[Y, N]_{A} \geqslant[Y, M]_{A}, Y \in \mathcal{I}(M)\right\}$.

Recall that a variety $\mathcal{X}$ is said to be unibranch if it is irreducible and the normalization map $\widetilde{\mathcal{X}} \rightarrow \mathcal{X}$ is bijective. Since any normalization map is closed, the above implies that $\widetilde{\mathcal{X}} \rightarrow \mathcal{X}$ is a homeomorphism. Hence unibranch varieties are topologically like normal varieties. An interesting question is whether $\overline{\mathcal{O}}_{M}$ is a normal variety if $\mathcal{I}(M)$ is a finite set.

The algebra $A$ is called representation finite if the set ind $A$ is finite. Then the set $\mathcal{I}(M)$ is finite as well, for any $M \in \bmod A$. Hence we derive the following consequence.

COROLlary 1.3. - Let $A$ be a representation finite algebra. Then the closure of any $\mathrm{Gl}_{d}(k)$-orbit in $\bmod _{A}^{d}(k)$ is a unibranch variety for any $d \geqslant 1$.

As another application of Theorem 1.2 we get the following result.

THEOREM 1.4. - If $M$ is a preprojective module in $\bmod A$ then $\mathcal{I}(M)$ is a finite set and hence the variety $\overline{\mathcal{O}}_{M}$ is unibranch.

We refer to Section 5 for the definition of preprojective modules.

The paper is organized as follows. In Section 2 we show a criterion for smooth points of the orbit closures in module varieties. Sections 3, 4 and 5 are devoted to the proofs of Theorems 1.1, 1.2 and 1.4, respectively. In Section 6 we present an example of an orbit closure in a module variety which is not unibranch. 


\section{Smooth points of orbit closures}

Let $M$ and $N$ be two modules in $\bmod A$. We shall use several times the characterization proved in [7] that the orbit $\mathcal{O}_{N}$ is contained in the closure $\overline{\mathcal{O}}_{M}$ of the orbit $\mathcal{O}_{M}$ if and only if there is an exact sequence in $\bmod A$ of the form

$$
0 \rightarrow Z \rightarrow Z \oplus M \rightarrow N \rightarrow 0
$$

for some module $Z$. Applying the left exact functor $\operatorname{Hom}_{A}(Y,-)$ to the above exact sequence we obtain the following well known fact.

Lemma 2.1. - Let $M, N, Y \in \bmod A$ and assume that $\mathcal{O}_{N} \subseteq \overline{\mathcal{O}}_{M}$. Then $[Y, M]_{A} \leqslant[Y, N]_{A}$.

The remainder of this section will be devoted to the proof of the following result.

Proposition 2.2. - Let $0 \rightarrow Z \rightarrow Z \oplus M \rightarrow N \rightarrow 0$ be an exact sequence in $\bmod A$ such that $[Z \oplus M, M]_{A}=[Z \oplus M, N]_{A}$. Then the variety $\overline{\mathcal{O}}_{M}$ is smooth at any point of the orbit $\mathcal{O}_{N}$.

Proof. - Let $\Omega: 0 \rightarrow Z \rightarrow Z \oplus M \stackrel{g}{\rightarrow} N \rightarrow 0$ be an exact sequence in $\bmod A$ such that

$$
[Z \oplus M, M]_{A}=[Z \oplus M, N]_{A} .
$$

We may assume that $N \in \bmod _{A}^{d}(k)$, where $d=\operatorname{dim}_{k} M=\operatorname{dim}_{k} N$. It suffices to show that the dimension of the tangent space $\mathcal{T}_{\overline{\mathcal{O}}_{M}, N}$ of $\overline{\mathcal{O}}_{M}$ at $N$ is not greater than $\operatorname{dim} \overline{\mathcal{O}}_{M}$. We have to recall some notation and results of Section 3 in [8]. Let

$$
\bmod _{A, Z \oplus M, t}^{d}:(\text { Commutative } k \text {-algebras }) \rightarrow(\text { Sets })
$$

be the functor defined in [8], Section 3.3, where $t=[Z \oplus M, M]_{A}$. This functor is represented by an algebraic $k$-scheme $\mathcal{X}$ such that the underlying variety is given by

$$
\mathcal{X}_{\text {red }}=\left\{L \in \bmod _{A}^{d}(k) ;[Z \oplus M, M]_{A}=[Z \oplus M, L]_{A}\right\} .
$$

In particular the orbits $\mathcal{O}_{M}$ and $\mathcal{O}_{N}$ are included in $\mathcal{X}_{\text {red }}$. Hence $\operatorname{dim}_{k} \mathcal{T}_{\overline{\mathcal{O}}_{M}, N} \leqslant \operatorname{dim}_{k} \mathcal{T}_{\mathcal{X}, N}$. On the other hand, the tangent space $\mathcal{T}_{\mathcal{X}, N}$ corresponds to the preimage of $N$ via the canonical map

$$
\bmod _{A, Z \oplus M, t}^{d}\left(k[\varepsilon] /\left(\varepsilon^{2}\right)\right) \rightarrow \bmod _{A, Z \oplus M, t}^{d}(k) .
$$

Let $U, V \in \bmod A$. The group of extensions $\operatorname{Ext}_{A}^{1}(V, U)$ may be interpreted as the quotient $\mathbb{Z}_{A}^{1}(V, U) / \mathbb{B}_{A}^{1}(V, U)$, where $\mathbb{Z}_{A}^{1}(V, U)$ is the group of cocycles, that is the $k$-linear maps $Z: A \rightarrow \operatorname{Hom}_{k}(V, U)$ satisfying

$$
Z\left(a a^{\prime}\right)=Z(a) V\left(a^{\prime}\right)+U(a) Z\left(a^{\prime}\right), \quad \text { for all } a, a^{\prime} \in A,
$$

and $\mathbb{B}_{A}^{1}(V, U)=\left\{h V-U h ; h \in \operatorname{Hom}_{k}(V, U)\right\}$ is the group of coboundaries. Any cocycle $Z$ in $\mathbb{Z}_{A}^{1}(V, U)$, where $U, V \in \bmod A$, induces an extension $W_{Z}$ of the $A$-module $V$ by the $A$-module $U$, which has the following block form

$$
W_{Z}=\left[\begin{array}{cc}
V & 0 \\
Z & U
\end{array}\right]
$$


Moreover, $W_{Z} \simeq V \oplus U$ if and only if $Z \in \mathbb{B}_{A}^{1}(V, U)$. Let $\mathbb{Z}_{A, L}^{1}(V, U)$ be the subset of $\mathbb{Z}_{A}^{1}(V, U)$ consisting of the cocycles $Z$ such that $\left[L, W_{Z}\right]_{A}=[L, U]_{A}+[L, V]_{A}$. Then $\mathbb{Z}_{A, L}^{1}(V, U)$ is a vector space containing $\mathbb{B}_{A}^{1}(V, U)$. Let $\operatorname{Ext}_{A, L}^{1}(V, U)=\mathbb{Z}_{A, L}^{1}(V, U) / \mathbb{B}_{A}^{1}(V, U)$ for any $U, V \in \bmod A$.

Applying Lemma 3.11 in [8] we get that

$$
\operatorname{dim}_{k} \mathcal{T}_{\mathcal{X}, N}=\operatorname{dim}_{k} \mathbb{Z}_{A, Z \oplus M}^{1}(N, N)
$$

Thus it remains to show that $\operatorname{dim}_{k} \mathbb{Z}_{A, Z \oplus M}^{1}(N, N) \leqslant \operatorname{dim} \overline{\mathcal{O}}_{M}$. Let $\operatorname{Aut}_{A}(M)$ denote the automorphism group of the $A$-module $M$. This is a nonempty open subset of the space of $A$-endomorphisms of $M$. Using the equalities

$$
\begin{aligned}
& \operatorname{dim} \overline{\mathcal{O}}_{M}=\operatorname{dim} \mathcal{O}_{M}=\operatorname{dim} \mathrm{Gl}_{d}(k)-\operatorname{dim} \operatorname{Aut}_{A}(M)=d^{2}-[M, M]_{A}, \\
& \operatorname{dim}_{k} \mathbb{Z}_{A, Z \oplus M}^{1}(N, N)=\operatorname{dim}_{k} \mathbb{B}_{A}^{1}(N, N)+\operatorname{dim}_{k} \operatorname{Ext}_{A, Z \oplus M}^{1}(N, N) \\
& =d^{2}-[N, N]_{A}+\operatorname{dim}_{k} \operatorname{Ext}_{A, Z \oplus M}^{1}(N, N),
\end{aligned}
$$

we reduce the problem to the inequality

$$
\operatorname{dim}_{k} \operatorname{Ext}_{A, Z \oplus M}^{1}(N, N) \leqslant[N, N]_{A}-[M, M]_{A} .
$$

If a short exact sequence $\Sigma: 0 \rightarrow N \rightarrow W \stackrel{g^{\prime}}{\rightarrow} N \rightarrow 0$ corresponds to an element $\xi$ of $\operatorname{Ext}_{A, Z \oplus M}^{1}(N, N)$ then the following induced sequence

$$
0 \rightarrow \operatorname{Hom}_{A}(Z \oplus M, N) \rightarrow \operatorname{Hom}_{A}(Z \oplus M, W) \rightarrow \operatorname{Hom}_{A}(Z \oplus M, N) \rightarrow 0
$$

is exact. It implies that $g: Z \oplus M \rightarrow N$ factors through $g^{\prime}: W \rightarrow N$. Hence, the pullback of $\Sigma$ via $g$ is a splittable exact sequence and consequently, $\xi$ belongs to the kernel of the last map in the following long exact sequence induced by $\Omega$ :

$$
\begin{aligned}
0 & \rightarrow \operatorname{Hom}_{A}(N, N) \rightarrow \operatorname{Hom}_{A}(Z \oplus M, N) \rightarrow \operatorname{Hom}_{A}(Z, N) \\
& \rightarrow \operatorname{Ext}_{A}^{1}(N, N) \rightarrow \operatorname{Ext}_{A}^{1}(Z \oplus M, N) .
\end{aligned}
$$

This implies that

$$
\operatorname{dim}_{k} \operatorname{Ext}_{A, Z \oplus M}^{1}(N, N) \leqslant[N, N]_{A}-[Z \oplus M, N]_{A}+[Z, N]_{A}=[N, N]_{A}-[M, N]_{A} .
$$

Since $[Z \oplus M, M]_{A}=[Z \oplus M, N]_{A}$, then $[M, M]_{A}=[M, N]_{A}$, by Lemma 2.1. Hence we obtain the inequality (2.1).

\section{Proof of Theorem 1.1}

Let $M, X \in \bmod A$ and $\mathcal{M}$ be the category of triples $(N, V, \varphi)$ such that $N \in \bmod A$, $V \in \bmod k$ and $\varphi: V \rightarrow \operatorname{Hom}_{A}(X, N)$ is a $k$-linear map. A homomorphism

$$
f:(N, V, \varphi) \rightarrow\left(N^{\prime}, V^{\prime}, \varphi^{\prime}\right)
$$


is a pair $f=\left(f_{1}, f_{2}\right)$ such that $f_{1} \in \operatorname{Hom}_{A}\left(N, N^{\prime}\right), f_{2} \in \operatorname{Hom}_{k}\left(V, V^{\prime}\right)$ and the following diagram

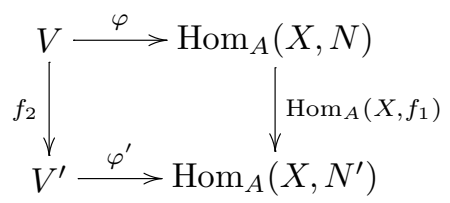

is commutative. Let $B$ be the one-point extension of $A$ by $X$, namely the algebra

$$
\left[\begin{array}{cc}
A & X \\
0 & k
\end{array}\right]
$$

with the usual addition and multiplication of matrices. If $(N, V, \varphi) \in \mathcal{M}$ then $N \oplus V$ becomes a $B$-module with the multiplication

$$
\left[\begin{array}{ll}
a & x \\
0 & \lambda
\end{array}\right] \cdot(n, v)=(a \cdot n+\varphi(v)(x), \lambda v) .
$$

One easily checks that this leads to an equivalence of categories

$$
\Phi: \mathcal{M} \rightarrow \bmod B
$$

(see for example Section 2.5 in [6]). We shall construct affine varieties corresponding to $\mathcal{M}$ like the module varieties $\bmod _{B}^{d}(k), d \geqslant 1$ correspond to $\bmod B$. Let $d \geqslant 1, c \geqslant 0$ and $\mathrm{Gl}_{(d, c)}(k)=\mathrm{Gl}_{d}(k) \times \mathrm{Gl}_{c}(k)$. Then

$$
\mathcal{D}=\bmod _{A}^{d}(k) \times \operatorname{Hom}_{k}\left(k^{c}, \operatorname{Hom}_{k}\left(X, k^{d}\right)\right)
$$

is an affine $\mathrm{Gl}_{(d, c)}(k)$-variety, where $(g, h) \star(N, \varphi)=\left(g \star N, \varphi^{\prime}\right)$ with $\varphi^{\prime}(v)(x)=g \varphi\left(h^{-1} v\right)(x)$ for any $v \in k^{c}$ and $x \in X$. We define $\mathcal{M}(d, c)$ as the closed $\mathrm{Gl}_{(d, c)}(k)$-invariant subset of $\mathcal{D}$ consisting of the pairs $(N, \varphi)$ such that $\operatorname{im} \varphi \subseteq \operatorname{Hom}_{A}(X, N)$, that is

$$
\varphi(v)(a x)=N(a)(\varphi(v)(x))
$$

for any $v \in k^{c}, a \in A$ and $x \in X$. Then the $\mathrm{Gl}_{d, c}(k)$-orbits in $\mathcal{M}(d, c)$ correspond bijectively to the isomorphism classes of triples $\widetilde{N}=(N, V, \varphi)$ in $\mathcal{M}$ with $\operatorname{dim}_{k} N=d$ and $\operatorname{dim}_{k} V=c$. We shall denote by $\mathcal{O}_{\widetilde{N}}$ the orbit in $\mathcal{M}(d, c)$ corresponding to $\widetilde{N}$.

Now we define the regular morphism $\Phi_{(d, c)}: \mathcal{M}(d, c) \rightarrow \bmod _{B}^{d+c}(k)$ in similar way as we have defined the equivalence $\Phi$. Let $(N, \varphi)$ belong to $\mathcal{M}(d, c)$ and $\xi$ denote the composition of the canonical isomorphisms:

$$
\operatorname{Hom}_{k}\left(k^{c}, \operatorname{Hom}_{k}\left(X, k^{d}\right)\right) \stackrel{\sim}{\rightarrow} \operatorname{Hom}_{k}\left(X, \operatorname{Hom}_{k}\left(k^{c}, k^{d}\right)\right) \stackrel{\sim}{\rightarrow} \operatorname{Hom}_{k}\left(X, \mathbb{M}_{d \times c}(k)\right) .
$$

Here and later on, $\mathbb{M}_{d \times c}(k)$ is the vector space of $d \times c$-matrices with coefficients in $k$. Then $\Phi_{(d, c)}(N, \varphi): B \rightarrow \mathbb{M}_{(d+c) \times(d+c)}(k)$ is the algebra homomorphism given by

$$
\Phi_{(d, c)}(N, \varphi)\left[\begin{array}{cc}
a & x \\
0 & \lambda
\end{array}\right]=\left[\begin{array}{cc}
N(a) & \xi(\varphi)(x) \\
0 & \lambda \cdot 1_{c}
\end{array}\right], \quad\left[\begin{array}{ll}
a & x \\
0 & \lambda
\end{array}\right] \in B
$$


The morphism $\Phi_{(d, c)}$ induces the bijection between the orbits in $\mathcal{M}(d, c)$ and some orbits in $\bmod _{B}^{d+c}(k)$, preserving and reflecting the closures and their geometric properties. In order to avoid introducing new notions, we formulate this fact in a less general form, which is sufficient for our applications.

Proposition 3.1. - Let $(N, \varphi)$ and $(P, \psi)$ be points of $\mathcal{M}(d, c)$. Then

$$
(N, \varphi) \in \overline{\mathrm{Gl}_{(d, c)}(k) \star(P, \psi)} \Leftrightarrow \Phi_{(d, c)}(N, \varphi) \in \overline{\mathrm{Gl}_{d+c}(k) \star \Phi_{(d, c)}(P, \psi)} .
$$

Furthermore, if this is the case, then $\overline{\mathrm{Gl}_{(d, c)}(k) \star(P, \psi)}$ is smooth at $(N, \varphi)$ if and only if $\overline{\mathrm{Gl}_{d+c}(k) \star \Phi_{(d, c)}(P, \psi)}$ is smooth at $\Phi_{(d, c)}(N, \varphi)$.

Proof. - Let $C=k e_{1} \times k e_{2}$ be the semisimple subalgebra of $B$, where $e_{1}=1_{A}$ and $e_{2}=1_{k}$ are orthogonal idempotents with $e_{1}+e_{2}=1_{B}$. The inclusion $C \subseteq B$ induces the $\mathrm{Gl}_{d+c}(k)$-equivariant regular morphism

$$
p: \bmod _{B}^{d+c}(k) \rightarrow \bmod _{C}^{d+c}(k)
$$

sending an algebra homomorphism $L: B \rightarrow \mathbb{M}_{d+c}(k)$ to its restriction $\left.L\right|_{C}: C \rightarrow \mathbb{M}_{d+c}(k)$. We denote by $E$ the element of $\bmod _{C}^{d+c}(k)$ given by

$$
E\left(\lambda_{1} \cdot e_{1}+\lambda_{2} \cdot e_{2}\right)=\left[\begin{array}{cc}
\lambda_{1} \cdot 1_{d} & 0 \\
0 & \lambda_{2} \cdot 1_{c}
\end{array}\right], \quad \lambda_{1}, \lambda_{2} \in k .
$$

The isotropy group of $E$ equals

$$
\left\{\left[\begin{array}{cc}
g & 0 \\
0 & h
\end{array}\right] \in \mathrm{Gl}_{d+c}(k) ; g \in \mathrm{Gl}_{d}(k), h \in \mathrm{Gl}_{c}(k)\right\}
$$

and hence $p^{-1}(E)$ is a $\mathrm{Gl}_{(d, c)}(k)$-variety. By Proposition 2 in [3], it suffices to show that $\operatorname{im} \Phi_{(d, c)}=p^{-1}(E)$ and the map $\Phi_{(d, c)}: \mathcal{M}(d, c) \rightarrow p^{-1}(E)$ is a $\operatorname{Gl}_{(d, c)}(k)$-equivariant isomorphism of varieties.

We claim that $p^{-1}(E)$ consists of the algebra homomorphisms $L: B \rightarrow \mathbb{M}_{d+c}(k)$ of the form

$$
L\left[\begin{array}{cc}
a & x \\
0 & \lambda
\end{array}\right]=\left[\begin{array}{cc}
N(a) & \psi(x) \\
0 & \lambda \cdot 1_{c}
\end{array}\right], \quad\left[\begin{array}{cc}
a & x \\
0 & \lambda
\end{array}\right] \in B
$$

for some maps $N: A \rightarrow \mathbb{M}_{d}(k)$ and $\psi: X \rightarrow \mathbb{M}_{d \times c}(k)$. Since $L$ is an algebra homomorphism then $N \in \bmod _{A}^{d}(k)$ and $\psi$ is a $k$-linear map satisfying $\psi(a x)=N(a) \psi(x)$ for any $a \in A$ and $x \in X$. Consequently, if $L$ is an algebra homomorphism of the form (3.1) then

$$
L\left(\lambda_{1} \cdot e_{1}+\lambda_{2} \cdot e_{2}\right)=\left[\begin{array}{cc}
N\left(\lambda_{1} \cdot 1_{A}\right) & \psi(0) \\
0 & \lambda_{2} \cdot 1_{c}
\end{array}\right]=\left[\begin{array}{cc}
\lambda_{1} \cdot 1_{d} & 0 \\
0 & \lambda_{2} \cdot 1_{c}
\end{array}\right]
$$

for any $\lambda_{1}, \lambda_{2} \in k$. This implies that $p(L)=E$.

Assume now that $L \in p^{-1}(E)$. Then

$$
E\left(e_{1}\right)=\left[\begin{array}{cc}
1_{d} & 0 \\
0 & 0
\end{array}\right] \text { and } E\left(e_{2}\right)=\left[\begin{array}{cc}
0 & 0 \\
0 & 1_{c}
\end{array}\right]
$$


Let $b=\left[\begin{array}{ll}a & x \\ 0 & \lambda\end{array}\right]$ be an element of $B$. Applying the algebra homomorphism $L$ to the equalities

$$
e_{1} \cdot b=\left[\begin{array}{cc}
a & x \\
0 & 0
\end{array}\right], \quad e_{2} \cdot b=\left[\begin{array}{cc}
0 & 0 \\
0 & \lambda
\end{array}\right], \quad b \cdot e_{1}=\left[\begin{array}{cc}
a & 0 \\
0 & 0
\end{array}\right] \quad \text { and } \quad b \cdot e_{2}=\left[\begin{array}{cc}
0 & x \\
0 & \lambda
\end{array}\right]
$$

we get that $L$ is of the form (3.1), which proves the claim.

From the above description of $p^{-1}(E)$ and the definition of $\Phi_{(d, c)}$ we obtain that $\operatorname{im} \Phi_{(d, c)} \subseteq p^{-1}(E)$. Let $(g, h) \in \mathrm{Gl}_{d, c}(k),(N, \varphi) \in \mathcal{M}(d, c),\left(N^{\prime}, \varphi^{\prime}\right)=(g, h) \star(N, \varphi), a \in A$, $x \in X$ and $\lambda \in k$. Then

$$
\begin{aligned}
\Phi_{(d, c)}((g, h) \star(N, \varphi))\left[\begin{array}{ll}
a & x \\
0 & \lambda
\end{array}\right] & =\Phi_{(d, c)}\left(N^{\prime}, \varphi^{\prime}\right)\left[\begin{array}{ll}
a & x \\
0 & \lambda
\end{array}\right]=\left[\begin{array}{cc}
N^{\prime}(a) & \xi\left(\varphi^{\prime}\right)(x) \\
0 & \lambda \cdot 1_{c}
\end{array}\right] \\
& =\left[\begin{array}{cc}
g N(a) g^{-1} & \xi\left(\varphi^{\prime}\right)(x) \\
0 & \lambda \cdot 1_{c}
\end{array}\right], \\
\left((g, h) \star \Phi_{(d, c)}(N, \varphi)\right)\left[\begin{array}{cc}
a & x \\
0 & \lambda
\end{array}\right] & =\left[\begin{array}{cc}
g & 0 \\
0 & h
\end{array}\right] \cdot\left[\begin{array}{cc}
N(a) & \xi(\varphi)(x) \\
0 & \lambda \cdot 1_{c}
\end{array}\right] \cdot\left[\begin{array}{ll}
g & 0 \\
0 & h
\end{array}\right]^{-1} \\
& =\left[\begin{array}{cc}
g N(a) g^{-1} & g(\xi(\varphi)(x)) h^{-1} \\
0 & \lambda \cdot 1_{c}
\end{array}\right] .
\end{aligned}
$$

From the definition of $\xi$ and $\varphi^{\prime}$ we conclude that

$$
\xi\left(\varphi^{\prime}\right)(x) v=\varphi^{\prime}(v)(x)=g \varphi\left(h^{-1} v\right)(x)=g \xi(\varphi)(x)\left(h^{-1} v\right)=\left(g \xi(\varphi)(x) h^{-1}\right) v
$$

for any $v \in k^{c}$. This implies that $\xi\left(\varphi^{\prime}\right)(x)=g \xi(\varphi)(x) h^{-1}$ and consequently, $\Phi_{(d, c)}$ is a $\mathrm{Gl}_{(d, c)}(k)$-equivariant morphism.

It suffices to show that there is a regular morphism $\eta: p^{-1}(E) \rightarrow \mathcal{M}(d, c)$ such that

$$
\eta \Phi_{(d, c)}(N, \varphi)=(N, \varphi) \quad \text { and } \quad \Phi_{(d, c)} \eta(L)=L
$$

for any $(N, \varphi) \in \mathcal{M}(d, c)$ and $L \in p^{-1}(E)$. We define $\eta$ as follows. If $L$ is an algebra homomorphism of the form (3.1) then $\eta(L)=\left(N, \xi^{-1}(\psi)\right)$. Since

$$
\xi^{-1}(\psi)(v)(a x)=\psi(a x) v=N(a) \psi(x) v=N(a)\left(\xi^{-1}(\psi)(v)(x)\right)
$$

for any $v \in k^{c}, a \in A$ and $x \in X$, the morphism $\eta$ is well defined. The proof of the equalities (3.2) is straightforward.

Proposition 3.2. - Let $\widetilde{N}, \widetilde{P} \in \mathcal{M}$ and $\Phi: \mathcal{M} \rightarrow \bmod B$ be the equivalence defined above. Then $\mathcal{O}_{\widetilde{N}} \subseteq \overline{\mathcal{O}}_{\widetilde{P}}$ if and only if $\mathcal{O}_{\Phi \widetilde{N}} \subseteq \overline{\mathcal{O}}_{\Phi \widetilde{P}}$. Furthermore, if this is the case, then $\overline{\mathcal{O}}_{\widetilde{P}}$ is smooth at any point of $\mathcal{O}_{\widetilde{N}}$ if and only if $\overline{\mathcal{O}}_{\Phi \widetilde{P}}$ is smooth at any point of $\mathcal{O}_{\Phi \widetilde{N}}$.

Proof. - Let $\widetilde{N}=(N, V, \varphi)$ and $\widetilde{P}=(P, W, \psi)$. The orbit $\mathcal{O}_{\widetilde{P}}$ is contained in $\mathcal{M}(d, c)$, where $d=\operatorname{dim}_{k} P$ and $c=\operatorname{dim}_{k} W$. Observe that the regular morphism $\Phi_{(d, c)}: \mathcal{M}(d, c) \rightarrow \bmod _{B}^{d+c}(k)$ is compatible with the equivalence $\Phi$, that is $\Phi_{(d, c)}\left(N^{\prime}, \varphi^{\prime}\right)$ belongs to the orbit $\mathcal{O}_{\Phi(\widetilde{T})}$ for any point $\left(N^{\prime}, \varphi^{\prime}\right)$ of the orbit $\mathcal{O}_{\widetilde{T}}$, where $\widetilde{T}=(T, U, \zeta)$ is an object of $\mathcal{M}$ with $\operatorname{dim}_{k} T=d$ and $\operatorname{dim}_{k} U=c$. Therefore the claim follows from Proposition 3.1 provided $\mathcal{O}_{\Phi \widetilde{N}}$ is contained in $\mathcal{M}(d, c)$.

If $\mathcal{O}_{\widetilde{N}} \subseteq \overline{\mathcal{O}}_{\widetilde{P}}$ then $\mathcal{O}_{\widetilde{N}} \subseteq \mathcal{M}(d, c)$. Assume that $\mathcal{O}_{\Phi \widetilde{N}} \subseteq \overline{\mathcal{O}}_{\Phi \widetilde{P}}$. It remains to show that $\operatorname{dim}_{k} N=d$ and $\operatorname{dim}_{k} V=c$. By the characterization of the orbit closures in $\bmod _{B}^{d+c}(k)$ 
mentioned in Section 2, there is an exact sequence in $\bmod B$ of the form

$$
0 \rightarrow Z \rightarrow Z \oplus \Phi \widetilde{P} \rightarrow \Phi \widetilde{N} \rightarrow 0
$$

for some $B$-module $Z$. Recall that $1_{B}=e_{1}+e_{2}$, where $e_{1}=1_{A}$ and $e_{2}=1_{k}$ are orthogonal idempotents. Then we get the exact sequences

$$
0 \rightarrow e_{i} Z \rightarrow e_{i} Z \oplus e_{i}(\Phi \widetilde{P}) \rightarrow e_{i}(\Phi \widetilde{N}) \rightarrow 0
$$

in $\bmod k$ for $i=1,2$. Hence,

$$
\begin{aligned}
& \operatorname{dim}_{k} N=\operatorname{dim}_{k} e_{1}(\Phi \widetilde{N})=\operatorname{dim}_{k} e_{1}(\Phi \widetilde{P})=\operatorname{dim}_{k} P=d, \\
& \operatorname{dim}_{k} V=\operatorname{dim}_{k} e_{2}(\Phi \widetilde{N})=\operatorname{dim}_{k} e_{2}(\Phi \widetilde{P})=\operatorname{dim}_{k} W=c,
\end{aligned}
$$

which finishes the proof.

Using the above proposition we can reformulate the results of Section 2. We abbreviate $\operatorname{dim}_{k} \operatorname{Hom}_{\mathcal{M}}(\widetilde{Y}, \widetilde{Z})$ by $[\widetilde{Y}, \widetilde{Z}]_{\mathcal{M}}$ for any objects $\widetilde{Y}$ and $\widetilde{Z}$ in $\mathcal{M}$.

COROLlary 3.3. - Let $\tilde{N}, \widetilde{P} \in \mathcal{M}$. Then $\mathcal{O}_{\widetilde{N}} \subseteq \overline{\mathcal{O}}_{\widetilde{P}}$ if and only if there is an exact sequence

$$
0 \rightarrow \widetilde{Z} \rightarrow \widetilde{Z} \oplus \widetilde{P} \rightarrow \widetilde{N} \rightarrow 0
$$

in $\mathcal{M}$ for some $\widetilde{Z}$. Furthermore, if this is the case, then:

(1) $[\widetilde{Y}, \widetilde{P}]_{\mathcal{M}} \leqslant[\widetilde{Y}, \widetilde{N}]_{\mathcal{M}}$ for any $\widetilde{Y} \in \mathcal{M}$.

(2) $\overline{\mathcal{O}}_{\widetilde{P}}$ is smooth at any point of $\mathcal{O}_{\widetilde{N}}$ provided $[\widetilde{Z} \oplus \widetilde{P}, \widetilde{P}]_{\mathcal{M}}=[\widetilde{Z} \oplus \widetilde{P}, \widetilde{N}]_{\mathcal{M}}$.

Let $\mathcal{F}: \bmod A \rightarrow \mathcal{M}$ be the full and faithful functor such that

$$
\mathcal{F} N=\left(N, \operatorname{Hom}_{A}(X, N), 1_{\operatorname{Hom}_{A}(X, N)}\right)
$$

for any module $N \in \bmod A$ and $\mathcal{F} \alpha=\left(\alpha, \operatorname{Hom}_{A}(X, \alpha)\right)$ for any $A$-homomorphism $\alpha: N \rightarrow N^{\prime}$.

Lemma 3.4. - Let $\widetilde{N}=(N, V, f)$ be an object in $\mathcal{M}$ such that $\mathcal{O}_{\widetilde{N}} \subseteq \overline{\mathcal{O}}_{\mathcal{F} M}$ and $f$ is injective. Then $[\mathcal{F} X, \mathcal{F} M]_{\mathcal{M}}=[\mathcal{F} X, \widetilde{N}]_{\mathcal{M}}$.

Proof. - The assumption $\mathcal{O}_{\widetilde{N}} \subseteq \overline{\mathcal{O}}_{\mathcal{F} M}$ implies that the orbits $\mathcal{O}_{\widetilde{N}}$ and $\mathcal{O}_{\mathcal{F} M}$ belong to the same variety $\mathcal{M}(d, c)$, where $d=\operatorname{dim}_{k} M$ and $c=[X, M]_{A}$. In particular, $\operatorname{dim}_{k} V=[X, M]_{A}$. Let $\alpha=\left(\alpha_{1}, \alpha_{2}\right)$ be a homomorphism in $\operatorname{Hom}_{\mathcal{M}}(\mathcal{F} X, \widetilde{N})$. Then we get the commutative diagram

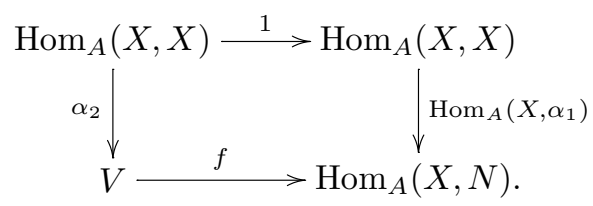

Since $f$ is injective, $\alpha_{2}$ is uniquely determined by $\alpha_{1}$. Furthermore, the image of $\operatorname{Hom}_{A}\left(X, \alpha_{1}\right)$ must be included in the image of $f$. In particular, $\alpha_{1}$ belongs to $\operatorname{im} f$. Since the functor $\mathcal{F}$ is full and faithful, then $[\mathcal{F} X, \mathcal{F} M]_{\mathcal{M}}=[X, M]_{A}$. Thus

$$
[\mathcal{F} X, \tilde{N}]_{\mathcal{M}} \leqslant \operatorname{dim}_{k} \operatorname{im} f=\operatorname{dim}_{k} V=[X, M]_{A}=[\mathcal{F} X, \mathcal{F} M]_{\mathcal{M}}
$$

The reverse inequality $[\mathcal{F} X, \mathcal{F} M]_{\mathcal{M}} \leqslant[\mathcal{F} X, \widetilde{N}]_{\mathcal{M}}$ follows from Corollary 3.3. 
Applying Corollary 3.3(1), Lemma 3.4 and the additivity of $\mathcal{F}$, we derive the following consequence.

Corollary 3.5. - Let $\widetilde{N}=(N, V, f) \in \mathcal{M}$. Assume that $\mathcal{O}_{\widetilde{N}} \subseteq \overline{\mathcal{O}}_{\mathcal{F} M}$ and $f$ is injective. Then $[\mathcal{F} L, \mathcal{F} M]_{\mathcal{M}}=[\mathcal{F} L, \widetilde{N}]_{\mathcal{M}}$ for any $L$ from $\operatorname{add}(X)$.

LEMma 3.6. - Let $\tilde{N}=(N, V, f)$ be an object of $\mathcal{M}$ such that $f$ is injective and $\mathcal{O}_{\widetilde{N}} \subseteq \overline{\mathcal{O}}_{\mathcal{F} M}$. Then there is an exact sequence

$$
0 \rightarrow Z \rightarrow Z \oplus M \stackrel{\beta}{\rightarrow} N \rightarrow 0
$$

in $\bmod A$ such that $\operatorname{im} f=\operatorname{im}_{\operatorname{Hom}_{A}}(X, \beta)$.

Proof. - Assume that $\mathcal{O}_{\widetilde{N}} \subseteq \overline{\mathcal{O}}_{\mathcal{F} M}$. Then there is an exact sequence

$$
0 \rightarrow \widetilde{Z} \stackrel{\gamma}{\rightarrow} \widetilde{Z} \oplus \mathcal{F} M \stackrel{\delta}{\rightarrow} \widetilde{N} \rightarrow 0
$$

in $\mathcal{M}$ for some $\widetilde{Z}=(Z, U, g), \gamma=\left(\gamma_{1}, \gamma_{2}\right)$ and $\delta=\left(\delta_{1}, \delta_{2}\right)$. This means that

$$
0 \rightarrow Z \stackrel{\gamma_{1}}{\rightarrow} Z \oplus M \stackrel{\delta_{1}}{\rightarrow} N \rightarrow 0
$$

is an exact sequence in $\bmod A$ and the diagram

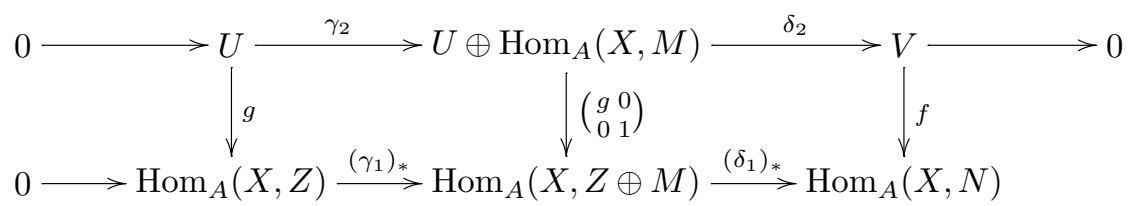

is commutative and has exact rows. Observe that $\operatorname{im} f \subseteq \operatorname{im}\left(\delta_{1}\right)_{*}$ and

$$
\operatorname{dim}_{k} \operatorname{im} f=\operatorname{dim}_{k} V=[X, M]_{A}=\operatorname{dim}_{k} \operatorname{im}\left(\delta_{1}\right)_{*} .
$$

This implies that $\operatorname{im} f=\operatorname{im}\left(\delta_{1}\right)_{*}=\operatorname{im}_{\operatorname{Hom}_{A}}\left(X, \delta_{1}\right)$.

Lemma 3.7. - Let $\tilde{N}=(N, V, f)$ be an object of $\mathcal{M}$ and $0 \rightarrow Z \stackrel{\alpha}{\rightarrow} Z \oplus M \stackrel{\beta}{\rightarrow} N \rightarrow 0$ an exact sequence in $\bmod A$ such that $\operatorname{im} f=\operatorname{im}_{\operatorname{Hom}_{A}}(X, \beta)$ and $f$ is injective. Then $\mathcal{O}_{\widetilde{N}} \subseteq \overline{\mathcal{O}}_{\mathcal{F} M}$. Furthermore, $\overline{\mathcal{O}}_{\mathcal{F} M}$ is smooth at any point of $\mathcal{O}_{\widetilde{N}}$ provided $Z \oplus M \in \operatorname{add}(X)$.

Proof. - Since $f: V \rightarrow \operatorname{Hom}_{A}(X, N)$ is an injective map, there is a unique map

$$
\beta^{\prime}: \operatorname{Hom}_{A}(X, Z \oplus M) \rightarrow V
$$

such that $\operatorname{Hom}_{A}(X, \beta)=f \beta^{\prime}$. Then we get the following commutative diagram with exact rows

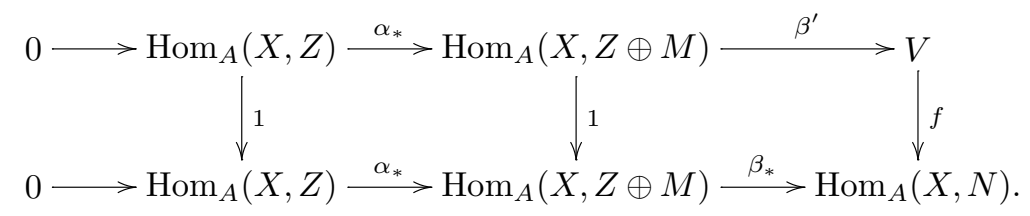


Since $\operatorname{dim}_{k} V=[X, M]_{A}$, we deduce that $\beta^{\prime}$ is surjective. Hence

$$
0 \rightarrow \mathcal{F} Z \stackrel{\mathcal{F} \alpha}{\longrightarrow} \mathcal{F}(Z \oplus M) \stackrel{\widetilde{\beta}}{\rightarrow} \widetilde{N} \rightarrow 0
$$

is an exact sequence in $\mathcal{M}$, where $\widetilde{\beta}=\left(\beta, \beta^{\prime}\right)$. Consequently, $\overline{\mathcal{O}}_{\mathcal{F} M}$ contains $\mathcal{O}_{\widetilde{N}}$, by Corollary 3.3 and since $\mathcal{F}(Z \oplus M)$ is isomorphic to $\mathcal{F} Z \oplus \mathcal{F} M$.

Assume that $Z \oplus M \in \operatorname{add}(X)$. Then $\overline{\mathcal{O}}_{\mathcal{F} M}$ is smooth at any point of $\mathcal{O}_{\widetilde{N}}$, by Corollaries 3.3 and 3.5.

Proof of Theorem 1.1. - Since Grassmann varieties are projective and $\overline{\mathcal{O}}_{M_{X}}$ is a closed subset of $\overline{\mathcal{O}}_{M} \times \operatorname{Grass}\left(\operatorname{Hom}_{k}\left(X, k^{d}\right), c\right)$, the morphism $p_{M, X}: \overline{\mathcal{O}}_{M_{X}} \rightarrow \overline{\mathcal{O}}_{M}$ is projective. Obviously $p_{M, X}$ is birational if its restriction $p^{\prime}: \mathcal{O}_{M_{X}} \rightarrow \mathcal{O}_{M}$ is an isomorphism. Let $\mathcal{H}$ be the subset of $\mathcal{O}_{M} \times \operatorname{Hom}_{k}\left(X, k^{d}\right)$ consisting of the pairs $\left(M^{\prime}, f\right)$ such that $f \in \operatorname{Hom}_{A}\left(X, M^{\prime}\right)$. Then the projection $q: \mathcal{H} \rightarrow \mathcal{O}_{M}$ is a vector subbundle of rank $c$ of the trivial bundle

$$
\mathcal{O}_{M} \times \operatorname{Hom}_{k}\left(X, k^{d}\right) \rightarrow \mathcal{O}_{M}
$$

(see Section 2.1 in [3] or Lemma 2.1 in [4]). This implies that $p^{\prime}$, being induced by $q$, is an isomorphism.

Recall that $\mathcal{M}(d, c)$ is a closed subset of the $\mathrm{Gl}_{(d, c)}(k)$-variety

$$
\mathcal{D}=\bmod _{A}^{d}(k) \times \operatorname{Hom}_{k}\left(k^{c}, \operatorname{Hom}_{k}\left(X, k^{d}\right)\right)
$$

and $\mathcal{O}_{M_{X}}$ is an orbit of the $\mathrm{Gl}_{d}(k)$-variety

$$
\mathcal{C}=\bmod _{A}^{d}(k) \times \operatorname{Grass}\left(\operatorname{Hom}_{k}\left(X, k^{d}\right), c\right) .
$$

Let $\mathcal{D}^{\prime}$ be the $\mathrm{Gl}_{(d, c)}(k)$-invariant open subset of $\mathcal{D}$ consisting of the pairs $(L, f)$ with $f$ injective. Then the map

$$
\pi: \mathcal{D}^{\prime} \rightarrow \mathcal{C}, \quad(L, f) \mapsto(L, \operatorname{im} f)
$$

is a principal $\mathrm{Gl}_{c}(k)$-bundle inducing a bijection between the sets of $\mathrm{Gl}_{(d, c)}(k)$-orbits in $\mathcal{D}^{\prime}$ and $\mathrm{Gl}_{d}(k)$-orbits in $\mathcal{C}$.

Let $\mathcal{C}_{A}$ be the subset of $\mathcal{C}$ consisting of the pairs $(L, V)$ such that $V \subseteq \operatorname{Hom}_{A}(X, L)$. Since $\pi$ is an open and surjective morphism and

$$
\pi^{-1}\left(\mathcal{C}_{A}\right)=\mathcal{D}^{\prime} \cap \mathcal{M}(d, c)
$$

is a closed subset of $\mathcal{D}^{\prime}$, then $\mathcal{C}_{A}$ is closed in $\mathcal{C}$. This implies that $\overline{\mathcal{O}}_{M_{X}}$ is contained in $\mathcal{C}_{A}$ and the induced map

$$
\pi^{\prime}: \mathcal{D}^{\prime} \cap \mathcal{M}(d, c) \rightarrow \mathcal{C}_{A}
$$

is again a principal $\mathrm{Gl}_{c}(k)$-bundle. Observe that $\pi^{\prime}\left(\mathcal{O}_{\mathcal{F} M}\right)=\mathcal{O}_{M_{X}}$. Then

$$
\left(\pi^{\prime}\right)^{-1}\left(\overline{\mathcal{O}}_{M_{X}}\right)=\overline{\mathcal{O}}_{\mathcal{F} M} \cap \mathcal{D}^{\prime}
$$

Since $\pi^{\prime}$ is a smooth morphism, the claim follows from Lemmas 3.6 and 3.7. 


\section{Proof of Theorem 1.2}

Throughout this section we assume that $\mathcal{I}(M)$ is a finite set. Denote by $\mathcal{S}(M)$ the full subcategory of $\bmod A$ consisting of the modules $Y$ such that there is an injective $A$-homomorphism $Y \rightarrow M^{i}$ for some $i \geqslant 1$. Obviously the category $\mathcal{S}(M)$ is closed under isomorphisms, submodules and direct sums.

Proof of the part (1). - Let $X$ be a module in $\bmod A$ such that $\mathcal{I}(M) \subseteq \operatorname{add}(X)$. Then any module from $\mathcal{S}(M)$ belongs to $\operatorname{add}(X)$. By Theorem 1.1, it remains to show that the variety $\overline{\mathcal{O}}_{M_{X}}$ is smooth. Let $(N, V)$ be a point of $\overline{\mathcal{O}}_{M_{X}}$. By Theorem 1.1, there is an exact sequence

$$
0 \rightarrow Z \stackrel{\alpha}{\rightarrow} Z \oplus M \stackrel{\beta}{\rightarrow} N \rightarrow 0
$$

in $\bmod A$ with $V=\operatorname{im}_{\operatorname{Hom}_{A}}(X, \beta)$. The above sequence has the form

$$
0 \rightarrow Z^{\prime} \oplus Z^{\prime \prime} \stackrel{\left(\begin{array}{cc}
1 Z^{\prime} & 0 \\
0 & \gamma \\
0 & \delta
\end{array}\right)}{\longrightarrow} Z^{\prime} \oplus Z^{\prime \prime} \oplus M \stackrel{(0, \varepsilon, \zeta)}{\longrightarrow} N \rightarrow 0,
$$

where $\gamma$ is a nilpotent endomorphism. Observe that the sequence

$$
0 \rightarrow Z^{\prime \prime} \stackrel{(\gamma, \delta)^{T}}{\longrightarrow} Z^{\prime \prime} \oplus M \stackrel{(\varepsilon, \zeta)}{\longrightarrow} N \rightarrow 0
$$

is exact and $\operatorname{im}_{H^{\prime}}(X, \beta)=\operatorname{im}_{A} \operatorname{Hom}_{A}(X,(\varepsilon, \zeta))$. By induction on $i$, we get that the homomorphism

$$
\left(\gamma^{i}, \delta \gamma^{i-1}, \delta \gamma^{i-2}, \ldots, \delta\right)^{T}: Z^{\prime \prime} \rightarrow Z^{\prime \prime} \oplus M^{i}
$$

is injective for any $i \geqslant 1$. We take $i$ such that $\gamma^{i}=0$. Then we obtain a monomorphism $Z^{\prime \prime} \rightarrow M^{i}$ which implies that $Z^{\prime \prime} \in \mathcal{S}(M)$. Consequently, $Z^{\prime \prime}$ belongs to $\operatorname{add}(X)$. Then $(N, V)$ is a smooth point of $\overline{\mathcal{O}}_{M_{X}}$, by Theorem 1.1 applied to the sequence (4.1).

We shall need the following lemma about unibranch varieties. This result was pointed out to the author by M. Reineke.

LEMMA 4.1. - Let $f: \mathcal{Y} \rightarrow \mathcal{X}$ be a proper birational morphism of irreducible varieties.

(1) If $\mathcal{X}$ is a unibranch variety then the fibres of $f$ are connected.

(2) If $\mathcal{Y}$ is a unibranch variety and the fibres of $f$ are connected then $\mathcal{X}$ is a unibranch variety as well.

Proof. - We take the normalization maps $g: \tilde{\mathcal{X}} \rightarrow \mathcal{X}$ and $h: \tilde{\mathcal{Y}} \rightarrow \mathcal{Y}$. Then we obtain a commutative diagram

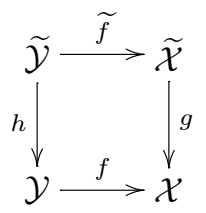

where $\tilde{f}$ is a proper birational morphism. Furthermore, the fibres of $\tilde{f}$ are connected, by the Zariski Main Theorem. 
Assume first that $\mathcal{X}$ is a unibranch variety. Then $g$ is a homeomorphism and hence $g \tilde{f}$ has connected fibres. Since $f h=g \widetilde{f}$ and $h$ is a surjective map, the fibres of $f$ are connected.

Assume now that $\mathcal{Y}$ is a unibranch variety, which implies that $h$ is a homeomorphism. If the fibres of $f$ are connected then the same holds for $g \widetilde{f}=f h$ and consequently for $g$ since $\tilde{f}$ is a surjective map. On the other hand, the map $g$ is finite, which implies that the fibres of $g$ are finite sets. Altogether we get that $g$ is a bijective map and $\mathcal{X}$ is a unibranch variety.

We may reduce the proof of parts (2) and (3) of Theorem 1.2 as follows. Assume that we have shown part (2) for some module $X \in \bmod A$ satisfying $\mathcal{I}(M) \subseteq \operatorname{add}(X)$. Then $p_{M, X}$ is a resolution of singularities. In particular, $\overline{\mathcal{O}}_{M_{X}}$ is a unibranch variety and we get part (3) of Theorem 1.2, by Lemma 4.1. Applying Theorem 1.1 and again Lemma 4.1, we obtain part (2) of Theorem 1.2, this time for arbitrary $X \in \bmod A$. Therefore we shall choose some special $A$-module $X$.

In the remainder of this section we assume that $X=\bigoplus_{Y \in \mathcal{I}(M)} Y$. Then $\mathcal{S}(M)=\operatorname{add}(X)$. In particular, $\mathcal{I}(M) \subseteq \operatorname{add}(X)$.

Let $Z \in \bmod A$ and $f_{1}, \ldots, f_{r}$ be a basis of $\operatorname{Hom}_{A}(Z, M)$. We define the $A$-homomorphism

$$
\varphi_{Z}=\left(f_{1}, \ldots, f_{r}\right)^{T}: Z \rightarrow M^{r} .
$$

Let $L_{Z}=\operatorname{ker} \varphi_{Z}$. Then $Z / L_{Z}$ belongs to $\mathcal{S}(M)$.

LeMmA 4.2. - Let $Y \in \mathcal{S}(M)$ and $Z \in \bmod A$. Then $[Z, Y]_{A}=\left[Z / L_{Z}, Y\right]_{A}$.

Proof. - The canonical surjective morphism $Z \rightarrow Z / L_{Z}$ induces an injective map $\operatorname{Hom}_{A}\left(Z / L_{Z}, Y\right) \rightarrow \operatorname{Hom}_{A}(Z, Y)$. Let $f \in \operatorname{Hom}_{A}(Z, Y)$. Since $Y \in \mathcal{S}(M)$, there is a monomorphism $g: Y \rightarrow M^{i}$ for some $i \geqslant 1$. Observe that any $A$-homomorphism $Z \rightarrow M^{i}$ factors through $\varphi_{Z}$ and hence

$$
\text { ker } f=\operatorname{ker} g f \subseteq \operatorname{ker} \varphi_{Z}=L_{Z} .
$$

Consequently, $f$ factors through $Z \rightarrow Z / L_{Z}$ which implies that the map

$$
\operatorname{Hom}_{A}\left(Z / L_{Z}, Y\right) \rightarrow \operatorname{Hom}_{A}(Z, Y)
$$

is also surjective.

Observe that the endomorphism algebra $E=\operatorname{End}_{A}(X)$ is basic, that is $E / \operatorname{rad} E$ is isomorphic to a product of copies of the field $k$. We have a canonical decomposition of the identity

$$
1_{E}=\sum_{Y \in \mathcal{I}(M)} e_{Y}
$$

into a sum of pairwise orthogonal primitive idempotents. For any module $N \in \bmod A$, the space $\operatorname{Hom}_{A}(X, N)$ has a natural (left) $E$-module structure. Since

$$
e_{Y} \cdot \operatorname{Hom}_{A}(X, N)=\operatorname{Hom}_{A}(Y, N)
$$

for any $Y \in \mathcal{I}(M)$, we obtain the formula for the dimension vector:

$$
\operatorname{dim}_{E} \operatorname{Hom}_{A}(X, N)=\left([Y, N]_{A}\right)_{Y \in \mathcal{I}(M)}
$$

Applying the generalization of the Auslander theorem proved in [2], we obtain the following result. 
COROLlary 4.3. - Let $U, V \in \mathcal{S}(M)$. Then $U \simeq V$ if and only if

$$
\operatorname{dim}_{E} \operatorname{Hom}_{A}(X, U)=\operatorname{dim}_{E} \operatorname{Hom}_{A}(X, V) .
$$

LEMMA 4.4. - Let $U$ and $V$ be modules in $\mathcal{S}(M)$ such that

$$
\operatorname{dim}_{E} \operatorname{Hom}_{A}(X, U)<\operatorname{dim}_{E} \operatorname{Hom}_{A}(X, V) .
$$

Then $[V, V]_{A}>[V, U]_{A}$.

Proof. - From the assumptions we conclude that $[W, U]_{A} \leqslant[W, V]_{A}$ for any $W \in \mathcal{S}(M)$ and $\left[Y^{\prime}, U\right]_{A}<\left[Y^{\prime}, V\right]_{A}$ for some $Y^{\prime} \in \mathcal{I}(M)$. Let $f_{1}, \ldots, f_{r}$ be a basis of $\operatorname{Hom}_{A}\left(Y^{\prime}, V\right)$ and consider the $A$-homomorphism

$$
\psi:\left(f_{1}, \ldots, f_{r}\right)^{T}: Y^{\prime} \rightarrow V^{r}
$$

Let $Z=\operatorname{coker} \psi$. Then the exact sequence $Y^{\prime} \stackrel{\psi}{\rightarrow} V^{r} \rightarrow Z \rightarrow 0$ in $\bmod A$ induces two exact sequences:

$$
\begin{aligned}
& 0 \rightarrow \operatorname{Hom}_{A}(Z, V) \rightarrow \operatorname{Hom}_{A}\left(V^{r}, V\right) \rightarrow \operatorname{Hom}_{A}\left(Y^{\prime}, V\right) \rightarrow 0 \\
& 0 \rightarrow \operatorname{Hom}_{A}(Z, U) \rightarrow \operatorname{Hom}_{A}\left(V^{r}, U\right) \rightarrow \operatorname{Hom}_{A}\left(Y^{\prime}, U\right) .
\end{aligned}
$$

By Lemma 4.2, we get

$$
\begin{aligned}
{\left[V^{r}, V\right]_{A} } & =[Z, V]_{A}+\left[Y^{\prime}, V\right]_{A}=\left[Z / L_{Z}, V\right]_{A}+\left[Y^{\prime}, V\right]_{A} \\
& >\left[Z / L_{Z}, U\right]_{A}+\left[Y^{\prime}, U\right]_{A}=[Z, U]_{A}+\left[Y^{\prime}, U\right]_{A} \geqslant\left[V^{r}, U\right]_{A} .
\end{aligned}
$$

Consequently, $[V, V]_{A}>[V, U]_{A}$.

Let $N \in \bmod A$. We define the subset $\mathcal{C}_{N}$ of $\operatorname{Grass}\left(\operatorname{Hom}_{A}(X, N), c\right)$ consisting of the subspaces $V$ of $\operatorname{Hom}_{A}(X, N)$ which are $E$-submodules with $\operatorname{dim}_{E} V=\operatorname{dim}_{E} \operatorname{Hom}_{A}(X, M)$. Recall that $c=[X, M]_{A}$.

LEMMA 4.5. - The set $\mathcal{C}_{N}$ is a (possibly empty) projective variety.

Proof. - Let $c_{Y}=[Y, M]_{A}$ for any $Y \in \mathcal{I}(M)$. Since

$$
\operatorname{Hom}_{A}(X, N)=\bigoplus_{Y \in \mathcal{I}(M)} \operatorname{Hom}_{A}(Y, N),
$$

the set

$$
\mathcal{D}=\coprod_{Y \in \mathcal{I}(M)} \operatorname{Grass}\left(\operatorname{Hom}_{A}(Y, N), c_{Y}\right)
$$

is a subvariety of $\operatorname{Grass}\left(\operatorname{Hom}_{A}(X, N), c\right)$. If $V \in \mathcal{C}_{N}$, then

$$
V=\bigoplus_{Y \in \mathcal{I}(M)} e_{Y} \cdot V
$$

where $e_{Y} \cdot V \subseteq \operatorname{Hom}_{A}(Y, N)$. This implies that $\mathcal{C}_{N}$ is a subset of $\mathcal{D}$. Furthermore, $\mathcal{C}_{N}$ is defined by the conditions

$$
\operatorname{Hom}_{A}(f, N)\left(e_{Y^{\prime}} \cdot V\right) \subseteq e_{Y} \cdot V
$$


for any $f \in \operatorname{Hom}_{A}\left(Y, Y^{\prime}\right)$ and $Y, Y^{\prime} \in \mathcal{I}(M)$. Thus $\mathcal{C}_{N}$ is a closed subset of the projective variety $\mathcal{D}$.

We shall need the following facts about projective $E$-modules. Their proofs are straightforward.

Lemma 4.6. - Any projective module in $\bmod E$ is isomorphic to $\operatorname{Hom}_{A}(X, W)$ for some $W \in \operatorname{add}(X)$. Moreover, the $k$-linear map

$$
\operatorname{Hom}_{A}(W, N) \rightarrow \operatorname{Hom}_{E}\left(\operatorname{Hom}_{A}(X, W) \operatorname{Hom}_{A}(X, N)\right)
$$

sending a homomorphism $f$ to $\operatorname{Hom}_{A}(X, f)$, is bijective for any $W \in \operatorname{add}(X)$ and $N \in \bmod A$.

Lemma 4.7. - Let $N \in \bmod A, V \in \mathcal{C}_{N}$ and assume that $\operatorname{dim}_{k} N=\operatorname{dim}_{k} M$. Then there is an exact sequence

$$
0 \rightarrow Z \rightarrow Z \oplus M \stackrel{\beta}{\rightarrow} N \rightarrow 0
$$

in $\bmod A$ such that $V=\operatorname{im}_{\operatorname{Hom}_{A}}(X, \beta)$.

Proof. - We consider a projective cover of the $E$-module $V$. By Lemma 4.6, we get a module $W \in \operatorname{add}(X)$ and a homomorphism $\gamma \in \operatorname{Hom}_{A}(W, N)$ such that $V$ is the image of the $E$-homomorphism

$$
\operatorname{Hom}_{A}(X, \gamma): \operatorname{Hom}_{A}(X, W) \rightarrow \operatorname{Hom}_{A}(X, N) .
$$

Let $Z=\operatorname{ker} \gamma$. Since $\operatorname{add}(X)=\mathcal{S}(M)$ and $\mathcal{S}(M)$ is closed under taking submodules, the modules $W$ and $Z$ belong to $\mathcal{S}(M)$. From the exact sequence of $E$-modules

$$
0 \rightarrow \operatorname{Hom}_{A}(X, Z) \rightarrow \operatorname{Hom}_{A}(X, W) \rightarrow V \rightarrow 0
$$

we conclude that

$$
\operatorname{dim}_{E} \operatorname{Hom}_{A}(X, W)=\operatorname{dim}_{E} \operatorname{Hom}_{A}(X, Z)+\operatorname{dim}_{E} V=\operatorname{dim}_{E} \operatorname{Hom}_{A}(X, Z \oplus M) .
$$

By Corollary 4.3, $W$ is isomorphic to $Z \oplus M$. Replacing $W$ by $Z \oplus M$ we obtain an exact sequence

$$
0 \rightarrow Z \rightarrow Z \oplus M \stackrel{\beta}{\rightarrow} N
$$

in $\bmod A$ with $V=\operatorname{im}_{\operatorname{Hom}_{A}}(X, \beta)$. Since $\operatorname{dim}_{k} N=\operatorname{dim}_{k} M$, the homomorphism $\beta$ is surjective.

LEMMA 4.8. - Let $N$ be a point of $\overline{\mathcal{O}}_{M}$. Then $p_{M, X}^{-1}(N)=\{N\} \times \mathcal{C}_{N}$.

Proof. - Let $(N, V) \in \overline{\mathcal{O}}_{M_{X}}$. Applying Theorem 1.1 we get an exact sequence

$$
0 \rightarrow Z \rightarrow Z \oplus M \stackrel{\beta}{\rightarrow} N \rightarrow 0
$$

in $\bmod A$ with $V=\operatorname{im}_{\operatorname{Hom}}(X, \beta)$. In particular, $V$ is an $E$-submodule of $\operatorname{Hom}_{A}(X, N)$. Furthermore, from the exact sequence of $E$-modules

$$
0 \rightarrow \operatorname{Hom}_{A}(X, Z) \rightarrow \operatorname{Hom}_{A}(X, Z \oplus M) \rightarrow V \rightarrow 0
$$

we obtain that $\operatorname{dim}_{E} V=\operatorname{dim}_{E} \operatorname{Hom}_{A}(X, M)$. Hence $V \in \mathcal{C}_{N}$. 
On the other hand, if $V \in \mathcal{C}_{N}$ then $(N, V) \in \overline{\mathcal{O}}_{M_{X}}$, by Theorem 1.1 and Lemma 4.7.

In order to prove that the variety $\mathcal{C}_{N}$ is connected for $N \in \overline{\mathcal{O}}_{M}$, we shall show that certain subvarieties of $\mathcal{C}_{N}$ are connected.

Proposition 4.9. - Let $N$ be a module in $\bmod A$ and $U$ an $E$-submodule of $\operatorname{Hom}_{A}(X, N)$ satisfying $\operatorname{dim}_{E} U \geqslant \operatorname{dim}_{E} \operatorname{Hom}_{A}(X, M)$. Then the subset

$$
\mathcal{C}_{U}=\left\{V \in \mathcal{C}_{N} ; V \subseteq U\right\}
$$

of $\mathcal{C}_{N}$ is nonempty and connected.

Proof. - Recall that $c=[X, M]_{A}$. We proceed by induction on $\operatorname{dim}_{k} U \geqslant c$. If $\operatorname{dim}_{k} U=c$ then $\operatorname{dim}_{E} U=\operatorname{dim}_{E} \operatorname{Hom}_{A}(X, M)$ and consequently, the variety $\mathcal{C}_{U}=\{U\}$ is nonempty and connected.

Assume that $\operatorname{dim}_{k} U>c$. Then $\operatorname{dim}_{E} U>\operatorname{dim}_{E} \operatorname{Hom}_{A}(X, M)$. As in the proof of Lemma 4.7, we consider a projective cover

$$
\pi: \operatorname{Hom}_{A}(X, W) \rightarrow U
$$

of the $E$-module $U$. This leads to an exact sequence

$$
0 \rightarrow Z \rightarrow W \stackrel{\beta}{\rightarrow} N
$$

such that $Z, W \in \mathcal{S}(M)$ and $U=\operatorname{im}_{\operatorname{Hom}_{A}}(X, \beta)$. From the exact sequence of $E$-modules

$$
0 \rightarrow \operatorname{Hom}_{A}(X, Z) \rightarrow \operatorname{Hom}_{A}(X, W) \rightarrow U \rightarrow 0
$$

we conclude that

$\operatorname{dim}_{E} \operatorname{Hom}_{A}(X, W)=\operatorname{dim}_{E} \operatorname{Hom}_{A}(X, Z)+\operatorname{dim}_{E} U>\operatorname{dim}_{E} \operatorname{Hom}_{A}(X, Z \oplus M)$.

Thus $[W, W]_{A}>[W, Z \oplus M]_{A}$, by Lemma 4.4. Let $s_{Y}$ denote the multiplicity of $Y$ as a direct summand of $W$, for any $Y \in \mathcal{I}(M)$. This means that $W$ is isomorphic to $\bigoplus_{Y \in \mathcal{I}(M)} Y^{s_{Y}}$. Then the set

$$
Q=\left\{Y \in \mathcal{I}(M) ; s_{Y}>0,[Y, W]_{A}>[Y, Z \oplus M]_{A}\right\}
$$

is not empty. Since $\pi$ is a projective cover, it induces an isomorphism of semisimple $E$-modules

$$
\operatorname{Hom}_{A}(X, W) / \operatorname{rad}_{E}\left(\operatorname{Hom}_{A}(X, W)\right) \simeq U / \operatorname{rad}_{E} U .
$$

This implies that the $E$-module $e_{Y} U / e_{Y} \operatorname{rad}_{E} U$ is isomorphic to $\left(S_{Y}\right)^{s_{Y}}$, where $S_{Y}=$ $\operatorname{Hom}_{A}(X, Y) / \operatorname{rad}_{E} \operatorname{Hom}_{A}(X, Y)$ is a simple $E$-module corresponding to the idempotent $e_{Y}$, for any $Y \in \mathcal{I}(M)$. Multiplying the sequence (4.2) by $e_{Y}$ we get the equality

$$
\operatorname{dim}_{k} e_{Y} U=[Y, W]_{A}-[Y, Z]_{A}
$$

for any $Y \in \mathcal{I}(M)$. Hence,

$$
Q=\left\{Y \in \mathcal{I}(M) ; e_{Y} \operatorname{rad}_{E} U \neq e_{Y} U, \operatorname{dim}_{k} e_{Y} U>[Y, M]_{A}\right\}
$$


Since $E$ is a basic algebra, we have that $\operatorname{dim}_{k} S_{Y}=1$ for any $Y \in \mathcal{I}(M)$ and there is a nice description of the maximal $E$-submodules of $U$. Namely, any such module is uniquely determined by $Y \in \mathcal{I}(M)$ with $e_{Y} \operatorname{rad}_{E} U \neq e_{Y} U$ and a codimension one $k$-subspace of $e_{Y} U$ containing $e_{Y} \operatorname{rad}_{E} U$.

Let $Y$ be an element of $\mathcal{I}(M)$ such that $e_{Y} \operatorname{rad}_{E} U \neq e_{Y} U$. Let $\mathcal{D}_{Y}$ be the subset of $\operatorname{Grass}\left(U, \operatorname{dim}_{k} U-1\right)$ consisting of the subspaces $P$ which are $E$-submodules of $U$ with $U / P \simeq S_{Y}$. Then $\mathcal{D}_{Y}$ is a projective variety isomorphic to

$$
\operatorname{Grass}\left(e_{Y} U / e_{Y} \operatorname{rad}_{E} U, s_{Y}-1\right) \simeq \mathbb{P}^{s_{Y}-1} .
$$

In particular, $\mathcal{D}_{Y}$ is an irreducible variety.

Assume that $V \in \mathcal{C}_{U}$. Then $V$ is a proper $E$-submodule of $U$. Hence $V$ is contained in a maximal $E$-submodule $P$ of $U$. Moreover, if $Y$ is an element of $\mathcal{I}(M)$ such that $U / P \simeq S_{Y}$, then

$$
\operatorname{dim}_{k} e_{Y} U>\operatorname{dim}_{k} e_{Y} P \geqslant \operatorname{dim}_{k} e_{Y} V=[Y, M]_{A}
$$

and hence $Y \in Q$. This implies that

$$
\mathcal{C}_{U}=\bigcup_{Y \in Q} \bigcup_{P \in \mathcal{D}_{Y}} \mathcal{C}_{P}
$$

Then $\mathcal{C}_{U}$ is nonempty, by the inductive assumptions and since $Q$ is nonempty as well as $\mathcal{D}_{Y}$ for any $Y \in Q$.

We consider now the closed subvariety

$$
\mathcal{E}_{Y}=\left\{(V, P) \in \mathcal{C}_{U} \times \mathcal{D}_{Y} ; V \subseteq P\right\}
$$

of $\mathcal{C}_{U} \times \mathcal{D}_{Y}$, for any $Y \in Q$. Let $q_{Y}: \mathcal{E}_{Y} \rightarrow \mathcal{D}_{Y}$ be the canonical projection for any $Y \in Q$. Since $\mathcal{C}_{U}$ is a closed subset of the projective variety $\mathcal{C}_{N}$, the map $q_{Y}$ is a projective morphism. Furthermore, the fibre $q^{-1}(P)=\mathcal{C}_{P} \times\{P\}$ is nonempty and connected for any $P \in \mathcal{D}_{Y}$, by the inductive assumptions. Since $q_{Y}$ is a surjective closed map with connected fibres and $\mathcal{D}_{Y}$ is a connected variety then $\mathcal{E}_{Y}$ is connected as well, for any $Y \in Q$. Let $\pi_{Y}: \mathcal{E}_{Y} \rightarrow \mathcal{C}_{U}$ denote the canonical projection for any $Y \in Q$. Then the morphism

$$
\left(\pi_{Y}\right)_{Y \in Q}: \coprod_{Y \in Q} \mathcal{E}_{Y} \rightarrow \mathcal{C}_{U}
$$

is surjective. Obviously $\operatorname{im} \pi_{Y}$ is connected for any $Y \in Q$.

Let $Y_{1}$ and $Y_{2}$ be two different elements of $Q$. It suffices to show that the set $\operatorname{im} \pi_{Y_{1}} \cap \operatorname{im} \pi_{Y_{2}}$ is not empty. We take $P_{1} \in \mathcal{D}_{Y_{1}}, P_{2} \in \mathcal{D}_{Y_{2}}$ and $P=P_{1} \cap P_{2}$. Then

$$
\operatorname{dim}_{E} P=\operatorname{dim}_{E} U-\operatorname{dim}_{E} S_{Y_{1}}-\operatorname{dim}_{E} S_{Y_{2}} \geqslant \operatorname{dim}_{E} \operatorname{Hom}_{A}(X, M) .
$$

Consequently, the set $\mathcal{C}_{P}$ is nonempty, by the inductive assumptions. We take $V \in \mathcal{C}_{P}$. Then $V$ belongs to $\mathcal{C}_{P_{i}}$ and hence $\left(V, P_{i}\right)$ belongs to $\mathcal{E}_{Y_{i}}$ for $i=1,2$. This implies that $V$ belongs to $\operatorname{im} \pi_{Y_{i}}$ for $i=1,2$.

Let $N$ be a point of $\overline{\mathcal{O}}_{M}$. Applying Lemma 4.8 and Proposition 4.9 for $U=\operatorname{Hom}_{A}(X, N)$, we get that the fibre $p_{M, X}^{-1}(N)$ is connected. This finishes the proof of parts (2) and (3) of Theorem 1.2. 
Proof of the part (4). - By Lemma 2.1, the variety $\overline{\mathcal{O}}_{M}$ is contained in the set

$$
R=\left\{N \in \bmod _{A}^{d}(k) ;[Y, N]_{A} \geqslant[Y, M]_{A}, Y \in \mathcal{I}(M)\right\} .
$$

Let $N$ be a point of $R$. Applying Proposition 4.9 for $U=\operatorname{Hom}_{A}(X, N)$ we get that the set $\mathcal{C}_{N}$ is nonempty. From Lemma 4.7 we get an exact sequence $0 \rightarrow Z \rightarrow Z \oplus M \rightarrow N \rightarrow 0$ in $\bmod A$, which implies that $N \in \overline{\mathcal{O}}_{M}$.

\section{Proof of Theorem 1.4}

Let $\left(\Gamma_{A}, \tau\right)$ denote the Auslander-Reiten quiver of the algebra $A$ (see [6] for details). We may identify the set of vertices of $\Gamma_{A}$ with ind $A$. A connected component $\mathcal{C}$ of $\Gamma_{A}$ is called preprojective if it has no cyclic paths and any $\tau$-orbit in $\mathcal{C}$ contains a projective module (see [6]). Moreover, a module from $\bmod A$ is said to be preprojective if it is isomorphic to a direct sum of modules from preprojective components. Dually, one defines preinjective modules, replacing projective modules by injective ones.

Let $M$ be a preprojective module in $\bmod A$. Thus we have a decomposition

$$
M \simeq \bigoplus_{1 \leqslant i \leqslant n}\left(M_{i}\right)^{b_{i}}
$$

such that $M_{i}$ is a vertex of a preprojective component $\mathcal{C}_{i}$ of $\Gamma_{A}$, for any $1 \leqslant i \leqslant n$. If $\left[X, M_{i}\right]_{A}>0$ then $X$ belongs to $\mathcal{C}_{i}$ (see 2.4(11) in [6]) and there is an oriented path in $\mathcal{C}_{i}$ leading from $X$ to $M_{i}$, for any $X \in$ ind $A$ and $1 \leqslant i \leqslant n$. The number of such vertices $X$ is finite and hence $\mathcal{I}(M)$ is a finite set. Consequently, the orbit closure $\overline{\mathcal{O}}_{M}$ is a unibranch variety, by Theorem 1.2.

Remark 5.1. - We would like to mention one more application of Theorem 1.2, which we shall not use. The notions and concepts from representation theory necessary to give a complete proof are quite involved; they can be found in [6]. Assume that $A$ is a tame concealed algebra (see [6] for the definition). Let $M$ be a module in $\bmod A$ having no nonzero preinjective direct summands. Then the set $\mathcal{I}(M)$ is finite and therefore the orbit closure $\overline{\mathcal{O}}_{M}$ is a unibranch variety. An interesting question is whether $\overline{\mathcal{O}}_{M}$ is unibranch for an arbitrary finite dimensional module $M$ over a tame concealed algebra.

\section{An example}

Let $A=k[X, Y] /\left(X^{2}, Y^{2}\right)$ and assume that the characteristic of $k$ is not equal to 2 . We give an example of an orbit closure in $\bmod _{A}^{4}(k)$ which is not unibranch. We view $\bmod _{A}^{d}(k)$ as the set of the pairs $L=\left(L_{X}, L_{Y}\right)$ of matrices in $\mathbb{M}_{d}(k)$ subjected to the conditions

$$
L_{X}^{2}=L_{Y}^{2}=L_{X} L_{Y}-L_{Y} L_{X}=0, \text { for any } d \geqslant 1 .
$$

Furthermore, the points of $\bmod _{A}^{d}(k)$ will be identified with the corresponding $d$-dimensional $A$-modules. We define

$$
M=\left(\left[\begin{array}{llll}
0 & 0 & 0 & 0 \\
1 & 0 & 0 & 0 \\
0 & 0 & 0 & 0 \\
0 & 0 & 1 & 0
\end{array}\right],\left[\begin{array}{llll}
0 & 0 & 0 & 0 \\
0 & 0 & 0 & 0 \\
1 & 0 & 0 & 0 \\
0 & 1 & 0 & 0
\end{array}\right]\right), \quad U_{\lambda}=\left(\left[\begin{array}{ll}
0 & 0 \\
1 & 0
\end{array}\right],\left[\begin{array}{ll}
0 & 0 \\
\lambda & 0
\end{array}\right]\right)
$$


for any $\lambda \in k$. Since there is an exact sequence in $\bmod A$ of the form

$$
\Sigma_{\lambda}: 0 \rightarrow U_{\lambda} \rightarrow M \rightarrow U_{-\lambda} \rightarrow 0
$$

the point

$$
N_{\lambda}=\left(\left[\begin{array}{llll}
0 & 0 & 0 & 0 \\
1 & 0 & 0 & 0 \\
0 & 0 & 0 & 0 \\
0 & 0 & 1 & 0
\end{array}\right],\left[\begin{array}{cccc}
0 & 0 & 0 & 0 \\
\lambda & 0 & 0 & 0 \\
0 & 0 & 0 & 0 \\
0 & 0 & -\lambda & 0
\end{array}\right]\right) \simeq U_{\lambda} \oplus U_{-\lambda}
$$

belongs to $\overline{\mathrm{Gl}_{4}(k) \star M}$, for any $\lambda \in k$.

Let $X$ be the unique, up to an isomorphism, simple $A$-module. Assume that $\lambda \neq 0$. We claim first that the fibre $p_{M, X}^{-1}\left(N_{\lambda}\right)$ contains at least two points. The exact sequences $\Sigma_{\lambda}$ and $\Sigma_{-\lambda}$ lead to the exact sequences:

$$
0 \rightarrow U_{\lambda} \rightarrow U_{\lambda} \oplus M \stackrel{\alpha}{\rightarrow} N_{\lambda} \rightarrow 0 \quad \text { and } \quad 0 \rightarrow U_{-\lambda} \rightarrow U_{-\lambda} \oplus M \stackrel{\beta}{\rightarrow} N_{\lambda} \rightarrow 0 .
$$

According to the equality $N_{\lambda}=U_{\lambda} \oplus U_{-\lambda}$, we have a decomposition

$$
\operatorname{Hom}_{A}\left(X, N_{\lambda}\right)=V \oplus V^{\prime}
$$

of $\operatorname{Hom}_{A}\left(X, N_{\lambda}\right)$ into a direct sum of two one-dimensional subspaces $V=\operatorname{Hom}_{A}\left(X, U_{\lambda}\right)$ and $V^{\prime}=\operatorname{Hom}_{A}\left(X, N_{\lambda}\right)$. Observe that $\operatorname{im}_{\operatorname{Hom}}(X, \alpha)=V$ and $\operatorname{im}_{\operatorname{Hom}}(X, \beta)=V^{\prime}$. Then $\left(N_{\lambda}, V\right)$ and $\left(N_{\lambda}, V^{\prime}\right)$ are different points of $p_{M, X}^{-1}\left(N_{\lambda}\right)$, by Theorem 1.1.

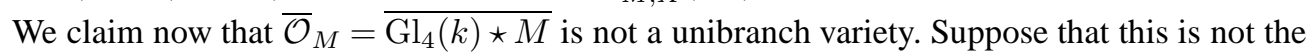
case. Let $\mathcal{U}$ be the union of orbits $\mathcal{O}_{N_{\lambda}}$ for all $\lambda \neq 0$. The fibres of $p_{M, X}: \overline{\mathcal{O}}_{M_{X}} \rightarrow \overline{\mathcal{O}}_{M}$ are connected, by Lemma 4.1. This implies that $\operatorname{dim} p_{M, X}^{-1}(N) \geqslant 1$ for any $N \in \mathcal{U}$. We need the following formula for the dimensions of orbits in module varieties, applied already in the proof of Proposition 2.2:

$$
\operatorname{dim} \mathcal{O}_{Y}=\operatorname{dim} \mathrm{Gl}_{d}(k)-[Y, Y]_{A},
$$

for any $Y \in \bmod _{A}^{d}(k)$ and $d \geqslant 1$. Then

$$
\operatorname{dim} \mathcal{O}_{M}=16-4=12 \text { and } \operatorname{dim} \mathcal{O}_{N_{\lambda}}=16-6=10,
$$

for any $\lambda \neq 0$. Observe that $\mathcal{O}_{N_{\lambda}}=\mathcal{O}_{N_{\mu}}$ if and only if $\lambda=\mu$ or $\lambda=-\mu$. This implies that $\operatorname{dim} \mathcal{U}=11$ and $\operatorname{dim} p_{M, X}^{-1}(\mathcal{U}) \geqslant 12$. Since $p_{M, X}$ is a birational map,

$$
\operatorname{dim} \overline{\mathcal{O}}_{M_{X}}=\operatorname{dim} \overline{\mathcal{O}}_{M}=12 \text { and } \operatorname{dim}\left(\overline{\mathcal{O}}_{M_{X}} \backslash \mathcal{O}_{M_{X}}\right) \leqslant 11
$$

On the other hand, $p_{M, X}^{-1}(\mathcal{U})$ is contained in $\overline{\mathcal{O}}_{M_{X}} \backslash \mathcal{O}_{M_{X}}$, a contradiction.

\section{Acknowledgements}

This article was written while I held a postdoc position at the University of Berne. I gratefully acknowledge support from the Schweizerischer Nationalfonds and Foundation for Polish Science. I would like to thank Christine Riedtmann for helpful comments during the preparation of this paper. 


\section{REFERENCES}

[1] Bobiński G., Zwara G., Normality of orbit closures for Dynkin quivers of type $\mathbb{A}_{n}$, Manuscr. Math. 105 (2001) 103-109.

[2] BongarTz K., A generalization of a theorem of M. Auslander, Bull. London Math. Soc. 21 (1989) 255-256.

[3] BongartZ K., Minimal singularities for representations of Dynkin quivers, Comment. Math. Helv. 63 (1994) 575-611.

[4] Bongartz K., On degenerations and extensions of finite dimensional modules, Advances Math. 121 (1996) 245-287.

[5] ReINEKE M., Quivers, desingularizations and canonical bases, Preprint, math.AG/0104284.

[6] Ringel C.M., Tame Algebras and Integral Quadratic Forms, in: Lecture Notes in Math., Vol. 1099, Springer-Verlag, 1984.

[7] Zwara G., Degenerations of finite dimensional modules are given by extensions, Compositio Math. 121 (2000) 205-218.

[8] ZWARA G., Smooth morphisms of module schemes, Proc. London Math. Soc. 84 (2002) 539-558.

(Manuscrit reçu le 29 juin 2001 ; accepté, après révision, le 3 décembre 2001.)

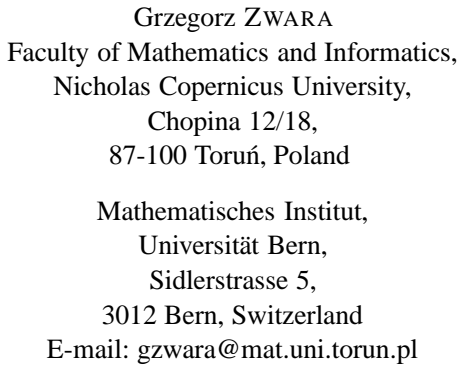

